\title{
Acceleration Signal Processing Method of Impact Response of Floating Shock Platform Based on Rigid Body Motion Model
}

\author{
Xiongliang Yao $\mathbb{D}$, Wenqi Zhang $\mathbb{D}$, Zhikai Wang $\mathbb{D}$, Jin Chen $\mathbb{D}$, and Heng Yang $\mathbb{E}$ \\ College of Shipbuilding Engineering, Harbin Engineering University, Harbin 150001, China \\ Correspondence should be addressed to Zhikai Wang; wangzhikai@hrbeu.edu.cn
}

Received 17 May 2020; Revised 6 September 2020; Accepted 19 October 2020; Published 29 October 2020

Academic Editor: Roberto Palma

Copyright (c) 2020 Xiongliang Yao et al. This is an open access article distributed under the Creative Commons Attribution License, which permits unrestricted use, distribution, and reproduction in any medium, provided the original work is properly cited.

\begin{abstract}
Floating shock platform is generally used to test the antishock performance of large shipboard equipment. Shock acceleration signal will produce zero-shift phenomenon in the test measurement process, which will affect the subsequent shock response spectrum analysis. In this paper, a method of shock acceleration signal processing based on rigid body motion revision model is established. The rigid body motion revision model adopts the theory of ship's seakeeping based on the hypothesis of KrylovFroude, in which the shock wave load of underwater explosion adopts the empirical formula. The bubble pulsation load adopts the GeersHunter spherical bubble model. The empirical mode decomposition method is used to eliminate the trend term of the low-frequency part of the acceleration signal, and the frequency filtering technology is used to eliminate the noise of the high frequency part. The response estimated by the rigid body motion model is used to modify the measured signal. The modified signal is analyzed by shock response spectrum to get the round design spectrum. The validity of the signal is determined by the Pauta criterion. Finally, the shock environment statistics of the whole platform is given. This method can eliminate the low-frequency trend term and high frequency noise and has good robustness. It can be applied to many kinds of signals. This method can provide technical support for antishock performance of shipboard equipment and also applied to other shock signal processing fields.
\end{abstract}

\section{Introduction}

Antishock assessment is required for shipborne equipment before it is fixed on a warship. For large shipborne equipment, floating shock platform (FSP) is generally used for antishock assessment. In the test process, the shock acceleration signal will inevitably produce zero-shift phenomenon which interferes with the low-frequency band of the signal. In order to accurately describe the actual shock response, it is necessary to eliminate the trend term of the measured acceleration signal; otherwise, the subsequent shock response spectrum (SRS) analysis cannot be carried out accurately.

Zhang et al. [1] studied the low-frequency shock response of the underwater explosion test of a 200 ton FSP and gave the low-frequency shock response characteristics of the FSP. In Zhang's study, the low-frequency shock response of the platform is studied by using the low-frequency vibrator. It is pointed out that the low-frequency shock response of different parts of the FSP are similar, indicating that the low-frequency response is mainly caused by rigid body motion. However, only the response data of the low-frequency vibrator is given in Zhang's study but not the response data of the platform and processing method.

The common methods to eliminate the trend term include least square fitting method [2], double exponential fitting method [3], wavelet transform method $[4,5]$, and empirical mode decomposition (EMD) method [6-8]. The double-exponential method fitting method is based on two piecewise exponential functions. The parameters of the function are obtained by fitting method, and the trend term is determined thereby. Both of wavelet transform method and EMD method decompose the signal into several components and determine the reconstructed signal through certain screening conditions. The screened component is the trend term. 
The basic idea of the above methods is to fit out the lowfrequency trend term components of acceleration signal and remove them all. But FSP will inevitably produce rigid body motion under the shock pressure, the rigidity of FSP is larger than warship, and its rigid body motion accounts for a larger proportion of the overall motion. If all the low-frequency components are considered as the errors introduced in the measurement process and eliminated, the response of the rigid body moving part will be lost, thus affecting the frequency response in the low-frequency range of SRS.

Based on the theory of plane wave and spherical wave, Sebert estimates the rigid body motion response of FSP under underwater explosion by using integral motion equation [9]. In Sebert's calculation, the shock wave first impacts the side of the FSP shell facing the explosion and then gradually moves away from the charge. The wave propagation under the shell accelerates the upward motion of FSP strip rolling. In both analysis and measurement, the deceleration is much less than the upward acceleration, as indicated by the slope of the velocity curve. High-frequency components of velocity time history are often observed in experiments. These frequencies are accompanied by vibration of inner bottom plate and bulwark, which are not considered in rigid body motion model. Sebert's calculation does not consider the effect of bubble pulsating load on FSP. In fact, even in the shallow water explosion condition in FSP test, the first expansion of bubble will produce low-frequency excitation on the platform, which will affect the rigid body motion of the platform to a certain extent. Furthermore, judging the validity of a measured data is also a very important problem in the test data processing, because it will directly affect the statistical analysis results of the overall shock environment of the platform.

In this paper, a method of shock acceleration signal processing is proposed based on rigid body motion revision model and EMD method, which judges the validity of the data by the Pauta criterion based on the design spectral displacement and velocity, so as to obtain the statistical analysis results of the shock environment in all directions of the platform. The practice shows that the method can eliminate the trend component in the signal and reflect the rigid motion response of the platform relatively accurately. It can also provide the basic support for the test and analysis of the impact resistance of shipborne equipment and can be used for reference in other areas of impact resistance.

\section{Materials and Methods}

\subsection{Rigid Motion Model of Floating Shock Platform}

2.1.1. Underwater Explosion Load. The underwater explosion load is mainly divided into shock wave load and bubble pulsation load. In this paper, the empirical formula [10] of Zamyshlyayev is used for the calculation of shock wave load:

$$
\begin{aligned}
P_{\text {shock }}= & P_{m} \times e^{-t / \theta}, \quad 0 \leq t<\theta, \\
P_{m}= & \begin{cases}4.41 \times 10^{7}\left(\frac{W^{1 / 3}}{R}\right)^{1.5}, & 6 \leq \frac{R}{R_{0}}<12, \\
5.24 \times 10^{7}\left(\frac{W^{1 / 3}}{R}\right)^{1.13}, & 12 \leq \frac{R}{R_{0}}<240,\end{cases} \\
\theta= & \begin{array}{ll}
0.45 R_{0}\left(\frac{R}{R_{0}}\right)^{0.45} \times 10^{-3}, & \frac{R}{R_{0}} \leq 30, \\
3.5 \frac{R_{0}}{c} \sqrt{\lg \frac{R}{R_{0}}-0.9,}, & \frac{R}{R_{0}} \geq 30,
\end{array}
\end{aligned}
$$

where $W$ is the mass of charge, $R$ is the distance between charge and test point, $c$ is the velocity of sound wave in water, and $R_{0}$ is the radius of charge.

The load in the bubble pulsation stage can be calculated by solving the G-H spherical bubble model $[11,12]$ :

$$
\begin{aligned}
\dot{a}= & \frac{\phi_{D}}{a}-c_{l}^{-1}\left(\dot{\phi}_{D}-\dot{a}^{2}-\frac{1}{3} \dot{u}^{2}-\frac{2}{3} \frac{\dot{u}}{a} \phi_{T}\right), \\
\dot{u}= & -2 \frac{\phi_{T}}{a}-c_{1}^{-1}\left(\dot{\phi}_{T}-2 \dot{a} \dot{u}\right), \\
\dot{\phi}_{D}= & \frac{1}{1+\zeta}\left[\left(\frac{1}{2}+\frac{1}{2} \frac{\rho_{g}}{\rho_{l}}+\zeta\right)\left(\dot{a}+\frac{1}{3} \dot{u}^{2}\right)\right. \\
& \left.-\frac{\rho_{g} c_{g}}{\rho_{l} a} \phi_{D}+\frac{2}{3 a}(1+\zeta) \dot{u} \phi_{T}-F\right], \\
\dot{\phi}_{T}= & \frac{1}{1+\zeta}\left[\left(1+\frac{\rho_{g}}{\rho_{l}}+2 \zeta\right) \dot{a} \dot{u}\right. \\
& \left.-\frac{\rho_{g} c_{g}}{\rho_{l} a}\left(2 \phi_{T}+\varphi_{T}\right)-\left(1-\frac{\rho_{g}}{\rho_{l}}\right) g a+\frac{3}{8} C_{D}|\dot{u}|^{E_{D}}\right],
\end{aligned}
$$

$F=\frac{P_{g}-P_{\mathrm{atm}}+\rho_{1} g(d-u)}{\rho_{1}}+\frac{1}{3}\left[\left(\frac{\phi_{T}}{a}\right)^{2}-\frac{\rho_{g}}{\rho_{l}}\left(\frac{\varphi_{T}}{a}\right)^{2}\right]$,

where $a(t)$ and $u(t)$ are the radius and vertical displacement of bubble, $\phi_{D}(t)$ and $\phi_{T}(t)$ are the expansion potential and migration potential of the fluid outside the bubble surface, $\varphi_{T}(t)$ is the migration potential of gas inside bubble surface, $c_{l}$ and $c_{g}$ are the sound speed in liquid and gas, $\rho_{g}(t)$ is the density of gas, $\zeta(t)=\rho_{g}(t) c_{g}(t) / \rho_{l} c_{l}, P_{g}(t)$ is the pressure of 
gas, $P_{\text {atm }}$ is the atmospheric pressure, $g$ is the acceleration of gravity, and $d$ is the depth of charge.

The pressure at any point in the flow field outside the bubble can be expressed as

$$
p_{\text {bubble }}=-\frac{\rho_{f}}{2}\left(a^{2} \cdot \dot{a} / R^{2}\right)^{2}+\frac{\rho_{f}}{R}\left[2 \dot{a}^{2} \cdot a+\ddot{a} \cdot a^{2}\right] .
$$

Simultaneous equations (2) to (7) are solved by the RungeKutta method with variable step sizes. The calculated bubble radius and its derivative are introduced into equation (8) to obtain the pressure in the bubble pulsation stage. So far, the pressure in shock wave stage and bubble stage of underwater explosion are solved completely, as shown in Figure 1.

It can be seen from Figure 1 that the peak pressure in shock wave stage is $1.2 \times 10^{7} \mathrm{~Pa}$, but its action time is short, which is millisecond level, while the peak pressure in bubble pulse stage is $0.5 \times 10^{6} \mathrm{~Pa}$ which is relatively small. But, its action time is long, which is second level. The effects of underwater explosion load on the platform are as follows: in the shock wave stage, the platform will produce large speed changes in a very short period of time and then continue to move due to the inertia; while in the bubble pulse stage, the load frequency is similar to the natural frequency of the rigid body motion of the platform, so the platform will produce large motion amplitude in this stage.

2.1.2. Rigid Body Motion Equation of Floating Shock Platform. According to the theory of ship seakeeping [13], the motion of floating body in waves meets the following differential equation:

$$
\begin{aligned}
M_{z z} \ddot{z}+2 N_{z z} \dot{z}+C_{z z} z & =F_{z z}, \\
M_{y y} \ddot{y}+2 N_{y y} \dot{y}+C_{y y} y & =F_{y y}, \\
M_{x x} \ddot{x}+2 N_{x x} \dot{x}+C_{x x} x & =F_{x x}, \\
M_{\varphi \varphi} \ddot{\varphi}+2 N_{\varphi \varphi} \dot{\varphi}+C_{\varphi \varphi} \varphi & =F_{\varphi \varphi}, \\
M_{\theta \theta} \ddot{\theta}+2 N_{\theta \theta} \dot{\theta}+C_{\theta \theta} \theta & =F_{\theta \theta}, \\
M_{\phi \phi} \ddot{\phi}+2 N_{\phi \phi} \dot{\phi}+C_{\phi \phi} \phi & =F_{\phi \phi},
\end{aligned}
$$

where $M, 2 N, C$, and $F$ represent the inertial force coefficient, damping force coefficient, restoring force coefficient, and wave force coefficient, and their lower angle scale represents the motion of each degree of freedom.

Square grids are divided on the wet surface of the floating shock platform, and the pressure $P_{i}$ at the center of grid and the normal direction $n_{i}$ outside the grid are calculated. In this paper, the wave disturbance force is calculated on the basis of KrylovFroude hypothesis, that is, the pressure field in waves is independent of the existence of ships. Considering only the mesh on the blasting surface, the propagation direction of the underwater explosion pressure wave is projected on the normal direction outside the mesh and then projected on each direction of the rigid body motion, and the sum of the fluid forces on all meshes can be used to obtain the wave disturbance dynamic coefficients of the floating shock platform in each direction of the rigid body motion under

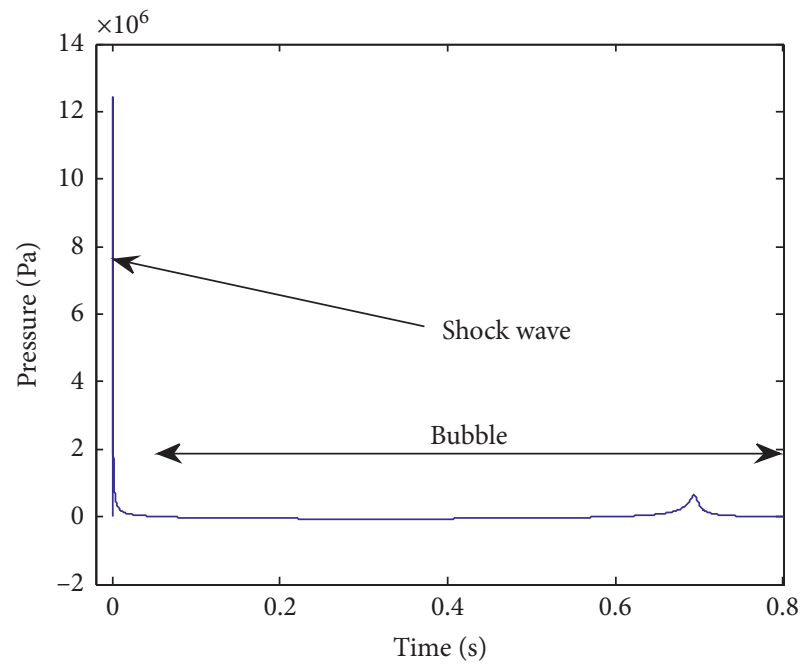

FIGURE 1: Time history curve of underwater explosion load.

the underwater explosion load. As the floating shock platform studied in this paper is a square floating body, the solution of its inertial force coefficient, damping force coefficient, and restoring force coefficient is relatively simple, which can refer to the theory of ship seakeeping and will not be discussed here. In addition, the influence of the sparse wave reflected by the free surface is not considered.

After the coefficients of the rigid body motion equations are solved, the central difference method can be used to solve the equations. Because the pressure changes rapidly in the shock wave stage, the time step of solution cannot be too large. In this paper, the discrete time step $\Delta t=5 e-4 \mathrm{~s}$ is used to solve the rigid body motion equation.

2.1.3. An Example of Rigid Body Motion. Considering the actual situation of the calibration test of the floating shock platform, the working conditions of the example are set as follows: the mass of the charge $W=55 \mathrm{~kg}$, the detonation distance $R=9.14 \mathrm{~m}$, the detonation depth $d=8.2 \mathrm{~m}$, the main dimension of the platform is $12.2 \times 6.1 \times 4.2 \mathrm{~m}$, and the mass of the platform body $M=100 \mathrm{~T}$. In this case, the rigid body motion of the platform and the flow field distribution in the fluid domain are shown in Figure 2:

As shown in Figure 2, when the spherical shock wave of $t=0.008 \mathrm{~s}$ acts on the platform, the platform begins to roll; when the bubble pulse pressure wave of $t=0.0755 \mathrm{~s}$ acts on the platform, the platform continues to roll due to the inertia and the bubble pulse pressure wave, the roll motion reaches the maximum value around $t=0.1805 \mathrm{~s}$, and then it begins to fall back. When $t=0.2480 \mathrm{~s}$, because of the continuous expansion and floating of bubbles, the upper surface of bubbles is close to the free surface, and the pressure wave generated will be significantly affected by the free surface. However, because the spherical bubble model is used in this paper, if the calculation is continued, the assumption of spherical bubble will not be satisfied. So, the total calculation time is $0.25 \mathrm{~s}$. 


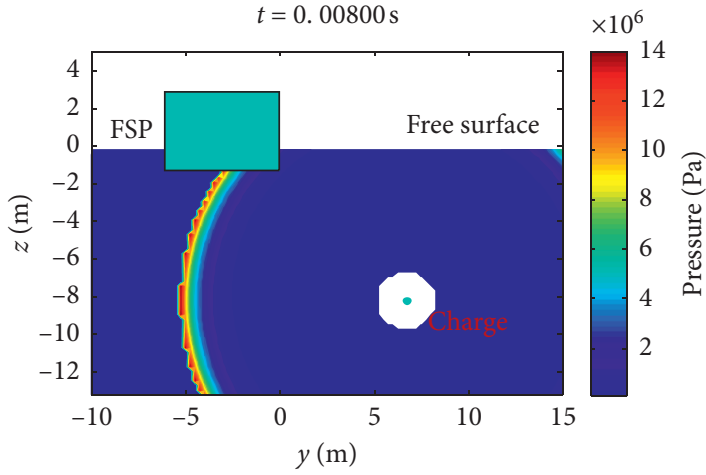

(a)

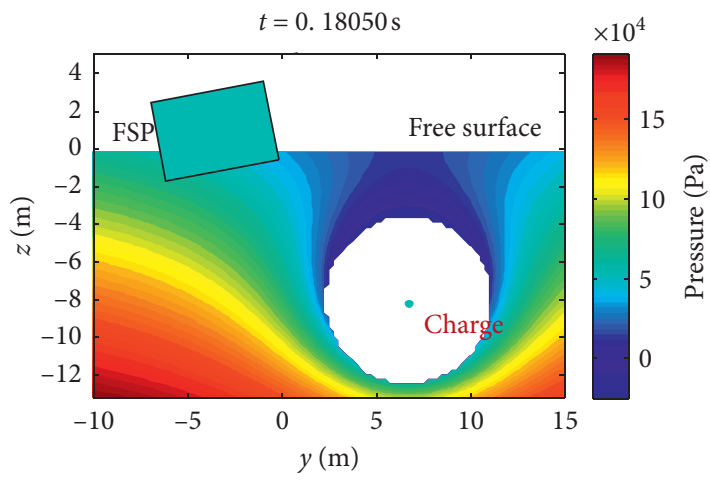

(c)

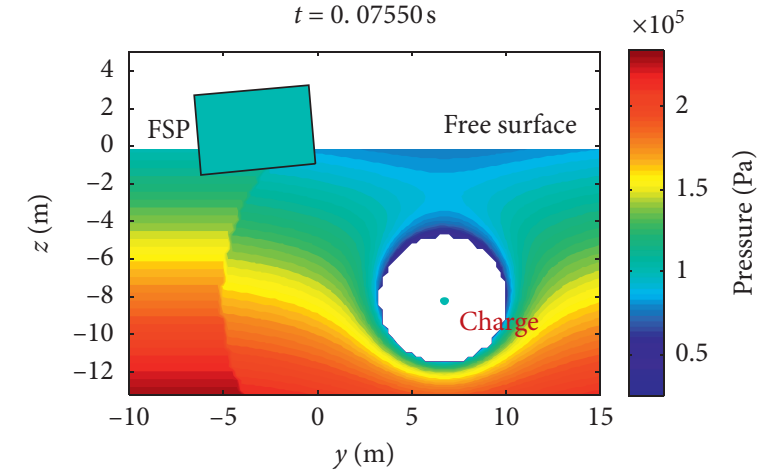

(b)

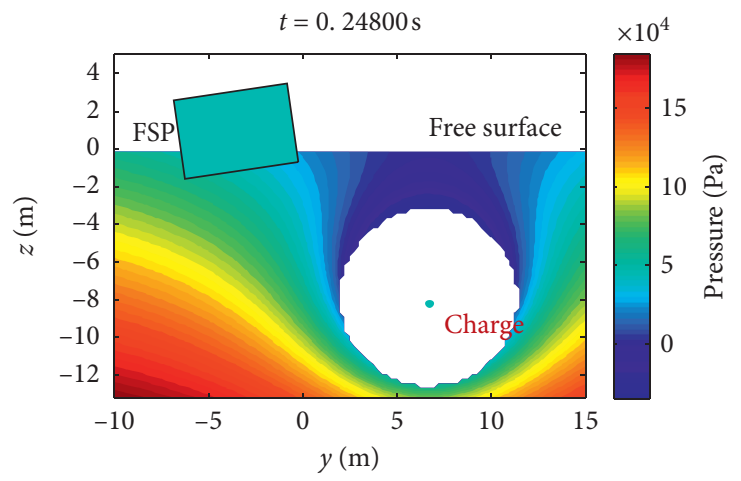

(d)

Figure 2: Rigid motion of platform under underwater explosion load. (a) $t=0.008 \mathrm{~s}$. (b) $t=0.0755 \mathrm{~s}$. (c) $t=0.1805 \mathrm{~s}$. (d) $t=0.2480 \mathrm{~s}$.

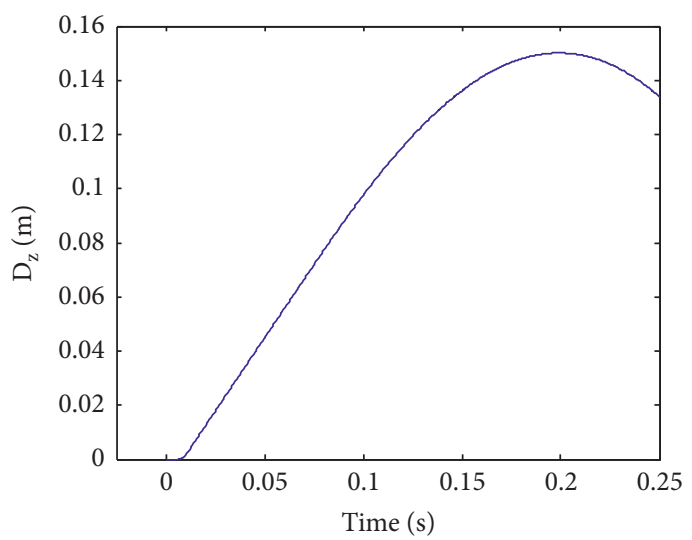

(a)

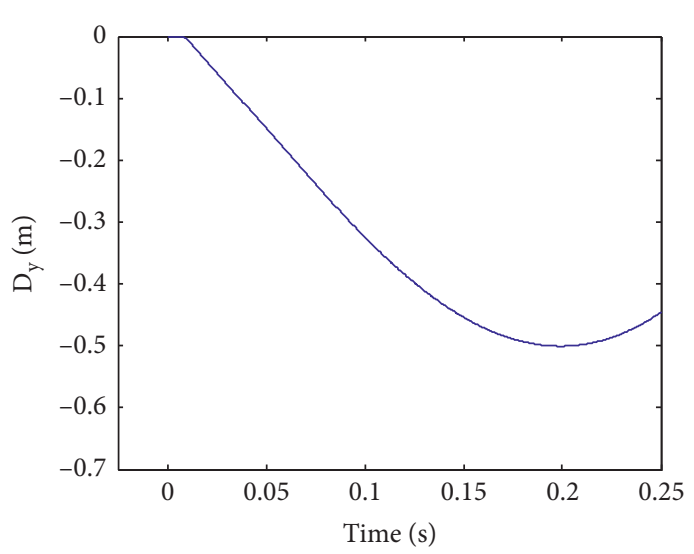

(c)

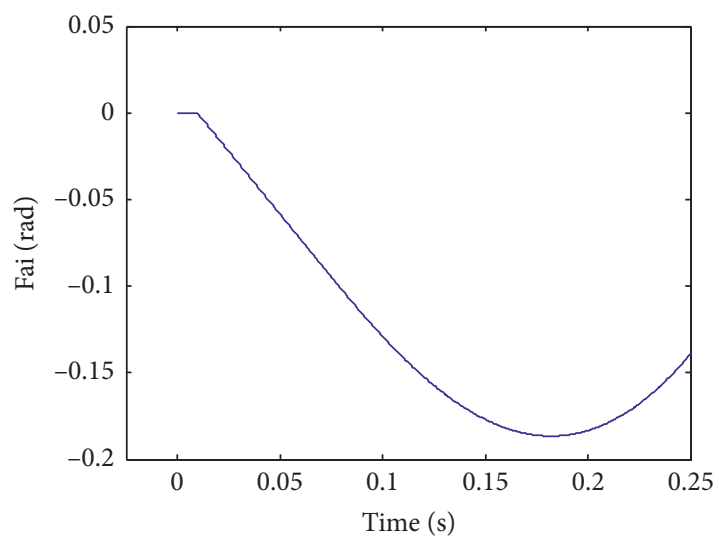

(b)

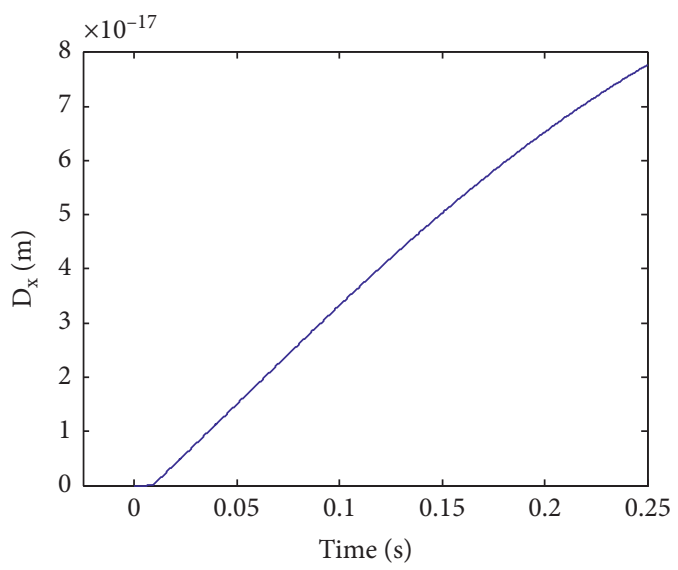

(d)

FIgURE 3: Rigid motion response of platform. (a) Heave. (b) Roll. (c) Sway. (d) Surge. 
The rigid body motion response of the platform is shown in Figure 3.

As shown in Figure 3, the platform mainly produces three directions of rigid body motion, namely, heave, roll, and sway. As the calculation condition is transverse condition, the position of the charge is symmetrical with respect to the cross section of the platform, the surge motion amplitude of the platform is very small, and the results shown in Figure 3(d) are errors resulting from numerical integration. The maximum platform heave movement displacement is $0.15 \mathrm{~m}$ at $0.1975 \mathrm{~s}$, the maximum angular displacement of platform rolling movement is $-0.1865 \mathrm{rad}$ at $0.1805 \mathrm{~s}$, and the maximum platform sway movement displacement is $-0.5006 \mathrm{~m}$ at $0.2 \mathrm{~s}$.

\subsection{Signal Processing Method of Shock Acceleration}

2.2.1. Truncation. As mentioned above, the underwater medium and far field explosion load is mainly divided into shock wave stage and bubble pulsation stage. The response signal of the structure can also be divided into shock wave stage response and bubble pulsation stage response according to the response characteristics. Du [14] et al. analyzed the structural response of a real ship in different stages of underwater explosion and pointed out that the acceleration amplitude of shock wave stage is high, the pulse width is narrow (about 1 millisecond), the acceleration amplitude of bubble pulse stage is low, and the pulse width is wide (several to more than 10 milliseconds). The Fourier transform of the response of shock wave stage and bubble pulse shows that the energy of shock wave stage is mainly high frequency (greater than $200 \mathrm{~Hz}$ ), and the energy of bubble pulse stage is mainly medium low frequency (less than $200 \mathrm{~Hz}$ ). However, the effect of acceleration response on shock spectrum in shock wave and bubble pulse stage is not discussed.

Zeng et al. [15] used acoustic solid coupling method to simulate the underwater noncontact explosion characteristics of a ship based on ABAQUS finite element analysis software, and on this basis, studied the shock environment characteristics of the underwater explosion bubble pulse pressure. The results show that the fluctuating pressure of bubbles can amplify the low frequency response of ships. When predicting the underwater explosion experiment and the shock environment, the more accurate shock environment data can be obtained when the calculation time is 1.5 times of the fluctuating period of bubbles.

The research above provides a certain theoretical support for the acceleration test signal processing of the floating shock platform, but there are some differences between the underwater explosion test of the floating shock platform and the warcraft. The underwater explosion test site of floating shock platform is generally shallow in water depth, the explosion depth of charge is about $10 \mathrm{~m}$, and the bubble will reach free surface in the first bubble pulsation period, while the explosion depth of warcraft test is tens of meters, and the bubble will generally pulse for multiple periods. In the following, take the G-H spherical bubble model as an example to calculate the bubble rising rule under typical working conditions of floating shock platform and warcraft, as shown in Table 1.

As shown in Figure 4, under the typical working condition of the FSP, due to the shallow initial explosion depth, the distance from the top surface of the bubble to the water surface in the first pulsation period is $2.71 \mathrm{~m}$, which is difficult to meet the spherical bubble assumption; however, under the typical working condition of the warcraft, due to the deep initial explosion depth, the distance from the bubble to the water surface after three pulsation periods is still large, regardless of the effect of the hull surface on the bubble under the influence, and the spherical bubble hypothesis can be satisfied.

In addition, the typical acceleration response of the inner bottom of the FSP measured by the test is shown in Figure 5 .

As shown in Figure 5, the acceleration response reaches the peak value $1.171 e 5 \mathrm{~m} / \mathrm{s}^{2}$ at $0.005 \mathrm{~s}$, while at $0.25 \mathrm{~s}$, the acceleration response is $337.3 \mathrm{~m} / \mathrm{s}^{2}$, which is $97.1 \%$ lower than the peak value. At $0.8 \mathrm{~s}$, the peak acceleration appears again with the value of $-133.1 \mathrm{~m} / \mathrm{s}^{2}$. The structure response in shock wave stage is much larger than that in bubble pulse stage. In conclusion, the following conclusions are drawn: when the shock environment of the FSP is studied through the test data, the influence of the bubble pulsating pressure on the acceleration response is not significant, and the cutoff time of the acceleration response is $0.25 \mathrm{~s}$.

\subsubsection{Acceleration Signal Preprocessing}

(1) Low-Pass Filtering. High-frequency noise is inevitable in the measurement of underwater explosion shock signal, and the noise will disturb the subsequent analysis and processing, so it must be eliminated. The purpose of eliminating high frequency noise can be realized by designing digital filter. According to the classification of mathematical operation, digital filtering includes frequency-domain filtering method and time-domain filtering method [2].

The frequency domain method use FFT algorithm to carry on the discrete Fourier transform to the input signal sampling data, analyze its frequency spectrum, according to the filtering requirements, set the frequency part which needs to be filtered directly to zero or add the gradual transition frequency band and then set to zero. Then IFFT algorithm is used to carry on the discrete Fourier inverse transform to the filtered data to recover the time domain signal. The frequency domain method has better frequency selectivity and flexibility, and its operation speed is much faster than the calculation of equivalent time domain convolution. Therefore, the frequency-domain method is more suitable for the processing of signals with large data length or with gradually decreasing vibration amplitude, such as shock response, seismic response, and other similar signals.

The time-domain method of digital filtering carries out the difference equation mathematical operation to the discrete data of signal to achieve the purpose of filtering. There are two methods to realize the classical digital filter: one is IIR digital filter, which is called infinite impulse response filter; the other is FIR filter, which is called finite impulse response filter. The equations of IIR and FIR filters are as follows: 
TABLE 1: The work condition of FSP and warcraft.

\begin{tabular}{lcc}
\hline & Mass of charge $(\mathrm{kg})$ & Depth of charge $(\mathrm{m})$ \\
\hline FSP & 55 & 8.2 \\
Warcraft & 1000 & 50 \\
\hline
\end{tabular}

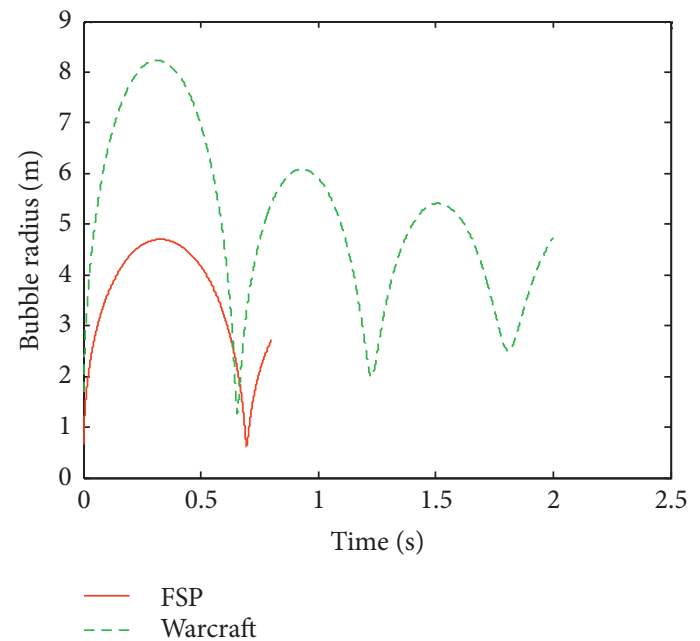

(a)

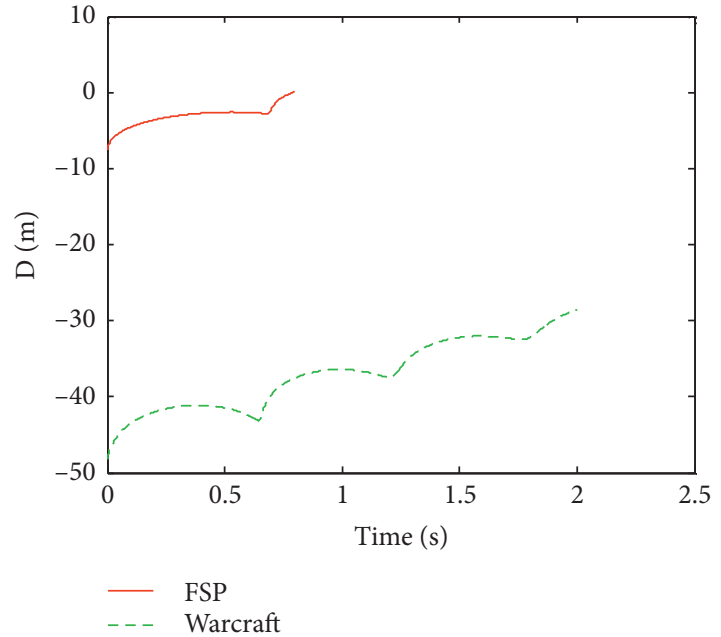

(b)

Figure 4: Comparison of bubble motion between FSP and warcraft under typical working conditions. (a) Bubble radius. (b) Distance from bubble top surface to free surface.

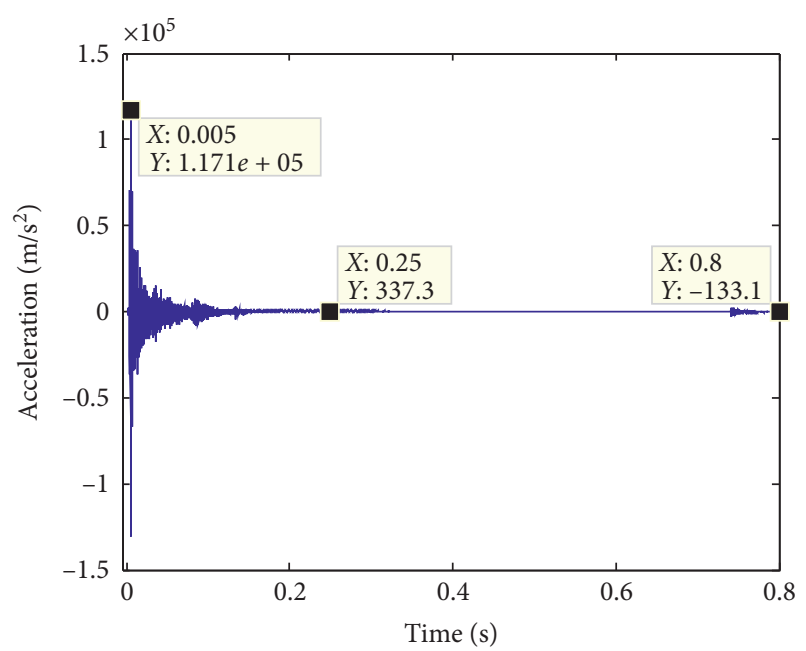

FIgURE 5: Typical acceleration response of inner bottom.

$$
\begin{aligned}
& y(n)=\sum_{k=0}^{N} a(k) x(n-k), \\
& y(n)=\sum_{k=0}^{N} a(k) x(n-k)+\sum_{j=0}^{P} b(j) y(n-j),
\end{aligned}
$$

where $x(n)$ is the input time series, $y(n)$ is the output time series, $a(k)$ is the coefficient of FIR filter, $N$ is the order of FIR filter, $a(k)$ and $b(j)$ are the coefficients of IIR filter, $N$ and $P$ are the order of IIR filter. Common IIR filters include Butterworth filter, Chebyshev I filter, Chebyshev II filter, and elliptical filter. According to the type and order of the filter, the coefficients of the filter can be obtained, and thus the filter output time series can be obtained.

In this paper, by comparing the effects of different filters on signal time and frequency domain, we can choose a suitable filter. All filters use $1000 \mathrm{~Hz}$ as cutoff frequency, and the order of IIR filter is 5 . The frequency-domain and timedomain effects of the filter are shown in Figures 6 and 7, respectively.

From Figure 6, we can clearly see the influence of different filters on the signal in the frequency domain. The cutoff frequency of all filters is $1000 \mathrm{~Hz}$, i.e., $0 \sim 1000 \mathrm{Hdz}$ is the passband of the filter. Among all the filters, Chebyshev I filter attenuates the signal on the passband, which is not ideal. Compared with the original signal, the energy of Chebyshev II filter on the stopband has the smallest attenuation, so it is not ideal. These two filters are excluded first. In addition, the transition band of frequency domain filtering is the shortest, and the energy of signal decays rapidly from $1000 \mathrm{~Hz}$ to a lower value in frequency domain.

It can be seen from Figure 7 that IIR filter (butter and ellip) will cause a certain time delay to the signal, but the frequency-domain method has no such phenomenon. The filtering effects of these three methods are not different in time domain except this phenomenon. Combining the filtering effect of frequency domain and time domain, it can be considered that frequency domain method is the most 
suitable filtering method for impulse signal. The comparison of the shock signal before and after filtering is shown in Figure 8.

As shown in Figure 8, the amplitude of the signal after frequency-domain filtering is reduced from $1.171 e 5 \mathrm{~m} / \mathrm{s}^{2}$ to $6.493 e 3 \mathrm{~m} / \mathrm{s}^{2}$, and the amplitude is reduced by $95 \%$. However, the decrease of signal amplitude is mainly caused by high-frequency noise signal, which has little effect on the response in the frequency range of tens to hundreds of $\mathrm{Hz}$ interested in shock spectrum analysis. In addition, through filtering, we can clearly see that the baseline of the signal deviates from the horizontal axis, that is, the so-called zerodrift phenomenon, and its trend component needs to be eliminated.

(2) Detrending. As mentioned above, in the measurement of underwater explosion shock signal, the measured data will appear zero drift and noise fluctuation, which may make the difference between the measured response signal and the real value, especially the response signal has a sudden low-frequency signal. The components of these noise signals mask the real and useful signals, thus affecting the analysis and calculation of shock response spectrum and the prediction of the shock environment which brings difficulties to the antishock design of the warcraft [3].

The methods of eliminating signal trend term mainly include high-pass filter method [16], least square method [17-19], wavelet transform method $[4,5,20]$, empirical mode decomposition method [6-8, 21-23], and double index correction method $[3,24]$. However, there are few studies on the elimination of the trend term of underwater explosion shock acceleration in the above literature. In this paper, based on the characteristics of underwater explosion shock acceleration, the corresponding method to eliminate the trend term is proposed.

The signal with uneliminated trend item of the typical measuring point is shown in Figure 9 It can be clearly seen from Figure 9 that the trend term component of the signal can be roughly divided into three segments in the time domain: the first segment has almost no obvious trend term component from the initial time to the maximum T0 time of the signal; the second segment has a rapid downward trend from the maximum T0 time to the minimum $\mathrm{T} 1$ time of the signal; the third segment has a rapid downward trend from the minimum Tl time of the signal from the moment to the end of the signal, the trend of the signal in this section shows a slow upward trend. Therefore, the signal can be processed in sections, and the trend items can be eliminated according to the different characteristics of each signal. Finally, the three signals can be synthesized to get the complete signal after eliminating the trend items.

In addition, the empirical mode decomposition method proposed by Huang [25] is used in the specific implementation method of eliminating trend item of each signal. Compared with the least square fitting method, the EMD method is more adaptive and can decompose the local characteristics of signals. In EMD, the decomposition process is based on a simple assumption that all signals are

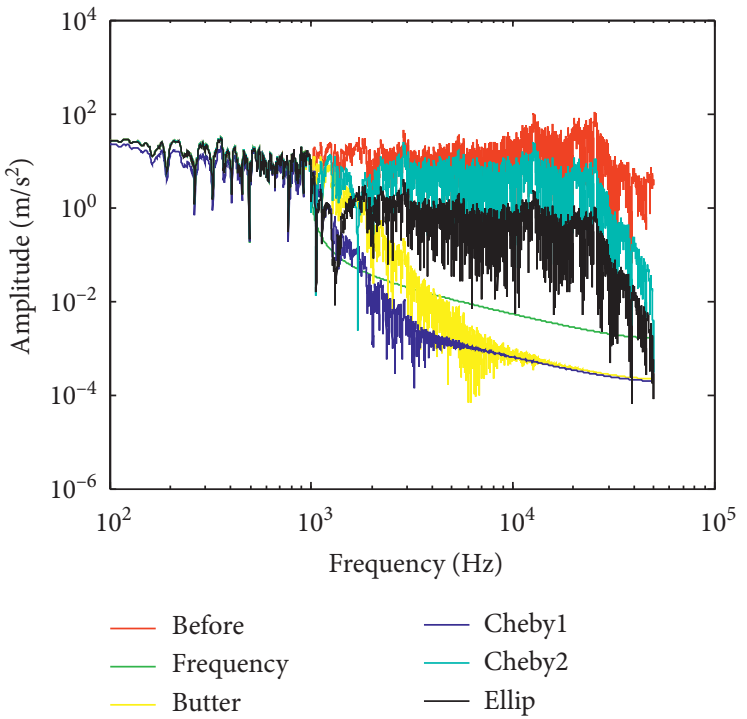

FIGURE 6: Frequency spectrum of signals filtered by different filters.

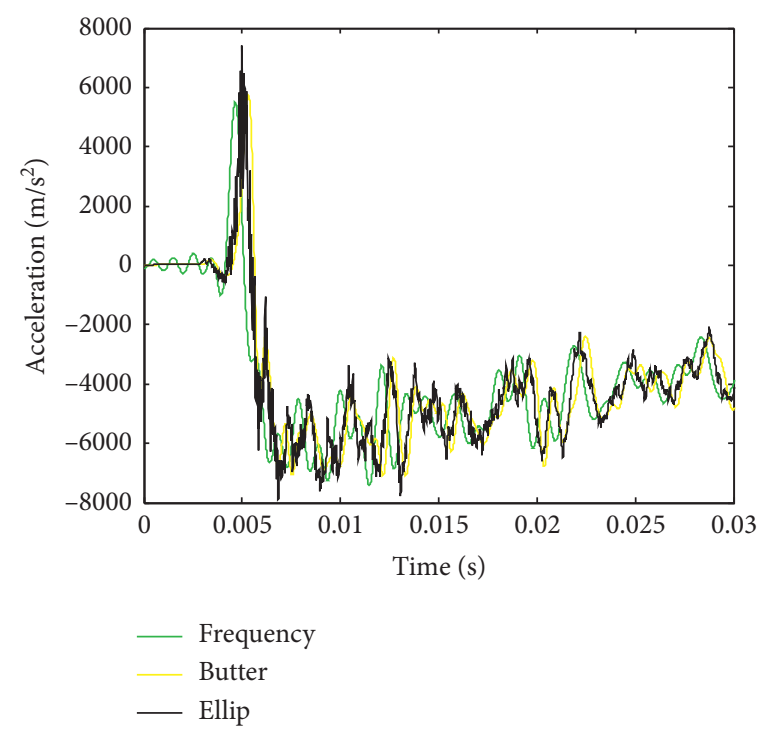

FIgURE 7: Time-history curve of signal filtered by different filters.

composed of different intrinsic mode functions (IMF). Each IMF component meets the following two restrictions:

(1) On the whole signal, the difference between the number of extreme points and the number of zero crossing points is not greater than 1

(2) At any point, the mean value of the upper and lower envelope is 0

The components of actual signals are complex and do not meet the above conditions. Therefore, Huang made the following assumptions:

(1) Any signal is composed of several IMF functions

(2) Each IMF can be either linear or nonlinear, with the same number of local zeros and extremum points of 

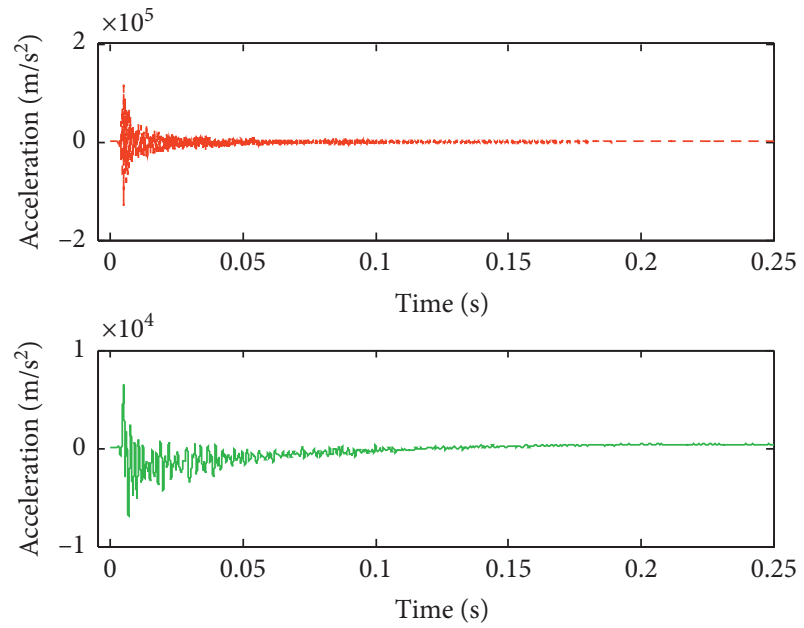

FIgURE 8: Signal comparison before and after filtering.

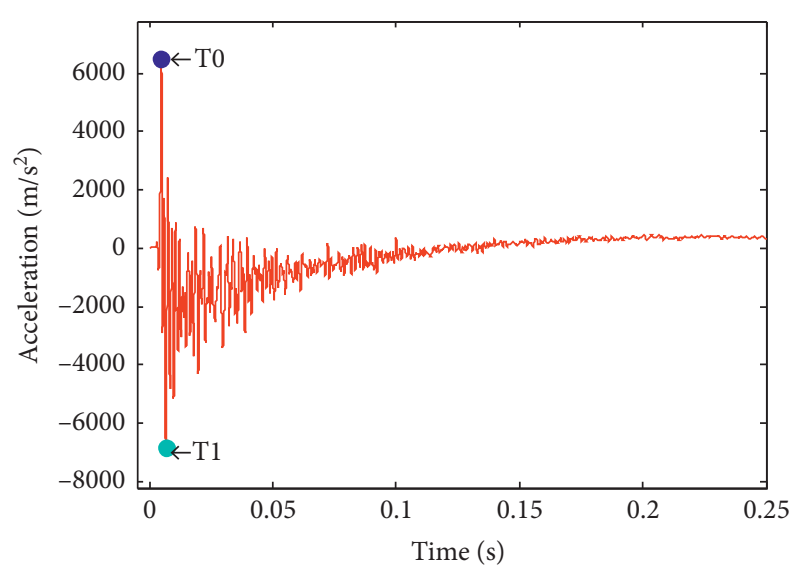

Figure 9: Signal of trend term not eliminated.

each IMF, and the upper and lower envelopes are locally symmetric with respect to the time axis

(3) At any time, a signal can contain several IMFs, if each modal function is overlapped with each other, which constitutes a composite signal

The flow chart of EMD decomposition signal is shown in Figure 10.

EMD decomposition of signal $s(t)$, a remainder $r$, and I IMF components are obtained, namely,

$$
s(t)=\sum_{n=1}^{I} c_{n}+r .
$$

According to the EMD decomposition principle, the IMF component is decomposed from high frequency to low frequency gradually, and to some extent, it satisfies the local symmetry. So, we can use these characteristics of IMF component to reconstruct the signal. In this paper, two thresholds, absolute mean value and maximum value, are set as the standard of signal reconstruction. Set different thresholds for the second and third signals to screen the IMF components and consider the IMF component that does not meet the threshold as the trend component, so as to achieve the elimination of the trend component processing based on the segmented EMD method.

It should also be noted that the above method cannot distinguish rigid body motion component of the signal from the trend component caused by the measurement error, because both of them are low-frequency components relative to the vibration of the elastomer in the signal. Therefore, the rigid body motion component in the signal is eliminated inevitably when the trend term is eliminated by segmented EMD. At this time, the signals processed above need to be superimposed on the rigid body motion components of the floating impact platform, that is, the calculation results described in Section 2. By integrating the measured acceleration signal with and without rigid body motion correction, the velocity signal can more clearly reflect the motion of the platform, as shown in Figure 11:

As shown in Figure 11, the velocity signal before correction reaches a stable value about $-2.5 \mathrm{~m} / \mathrm{s}$ at about $0.05 \mathrm{~s}$ and then fluctuates gradually up and down, which is not in line with the actual situation. As shown in the calculation results of the rigid body motion model, the FSP will move under the combined action of the bubble load and the static load after the shock wave phase. The EMD method is used to eliminate the trend term in the original signal, and the rigid body motion response in the low-frequency part is also eliminated. The modified velocity signal can better reflect the motion characteristics of FSP, so it can be considered that the modified signal is more consistent with the actual situation.

\subsubsection{Spectrum Analysis}

(3) Fourier Spectrum Analysis. Fourier spectrum analysis is one of the most common spectrum analysis methods. The measured acceleration shock signal belongs to digital signal, which can be converted into frequency domain signal by FFT algorithm. The main feature of FFT is to greatly reduce the number of operations required for DFT [2]. At the same time, due to the reduction of the number of operations, the operation time is reduced and the use efficiency of the computer is improved. Due to the high efficiency of FFT, Fourier spectrum analysis can be used to observe the frequency characteristics of the signal after the shock signal preprocessing. The Fourier spectrum comparison before and after the typical shock signal preprocessing is shown in Figure 12.

As shown in Figure 12, the energy in the low frequency $(1-100 \mathrm{~Hz})$ of the signal is greatly reduced after eliminating the trend term processing, which further proves the effectiveness of the trend term processing; the energy in the if middle frequency part $(100 \mathrm{~Hz}-1000 \mathrm{~Hz})$ of the signal has almost no change, indicating that the signal preprocessing does not lose the effective component of the signal; the energy in the high frequency part (above $1000 \mathrm{~Hz}$ ) of the signal also has reduced to a great extent, the reduction is due to the low-pass filter processing in the frequency domain. 


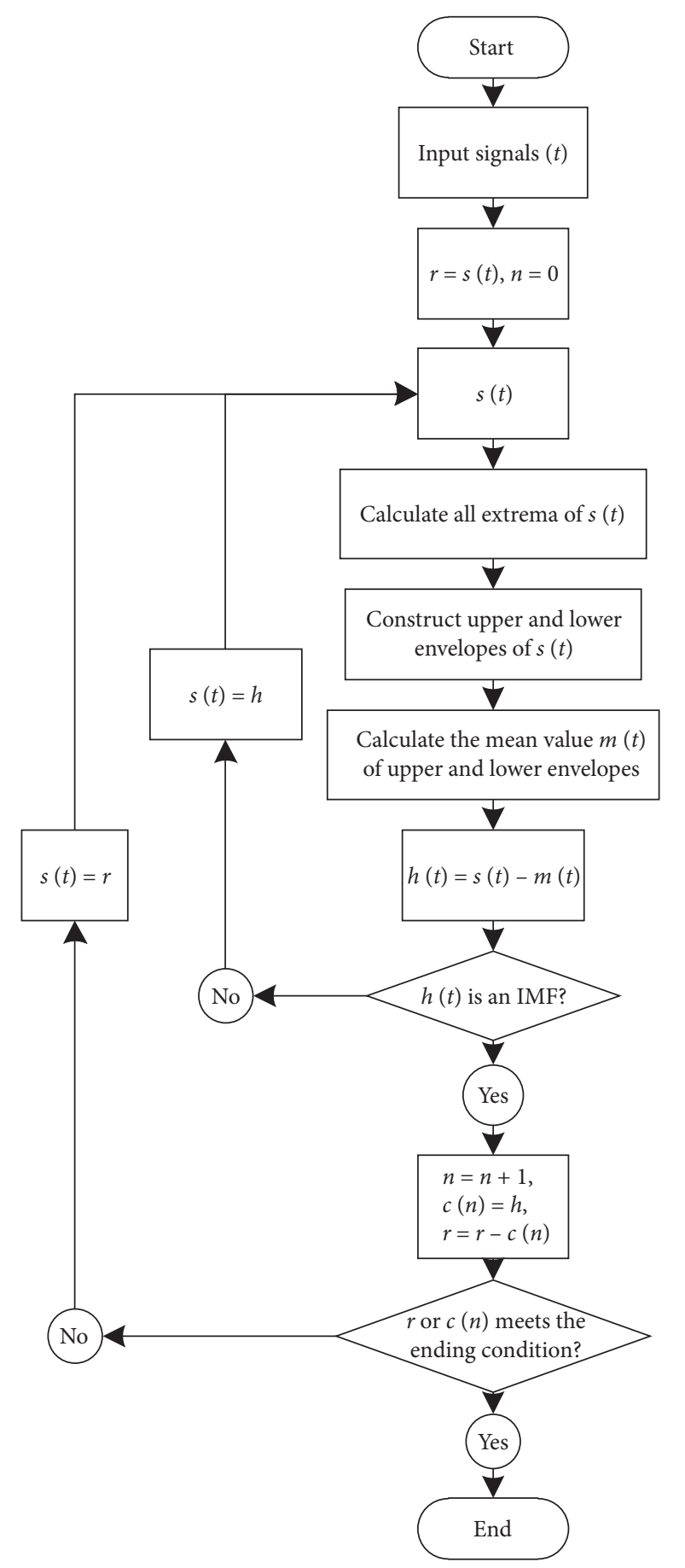

Figure 10: The flow chart of EMD method after EMD decomposition of signal $s(t)$, a remainder $r$, and I IMF components.

(4) Shock Response Spectrum Analysis. The shock response spectrum was first proposed by Maurice Biot in 1932. After a long time of development, it has been widely used in the fields of earthquake engineering, aerospace, and ship shock resistance [26]. Generally, acceleration time-history data are used to describe the frequency response of impact environment and to evaluate the maximum value of structural dynamic response. Assuming that a series of single degree of

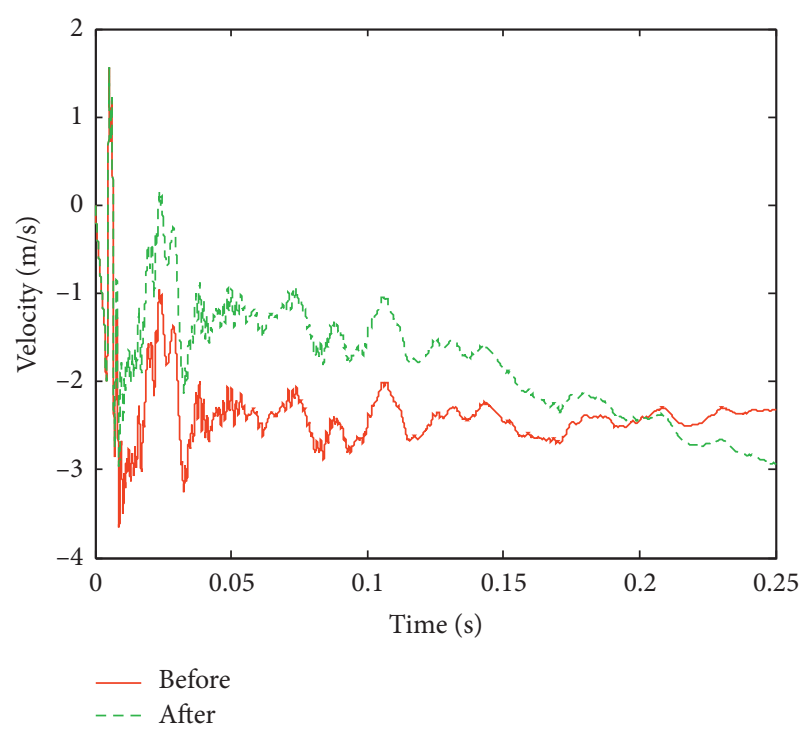

FIGURE 11: Comparison of velocity signals before and after rigid body motion correction.

freedom spring oscillators are installed on the foundation, the natural frequencies of spring oscillators are $f_{1}, f_{2}, f_{3}, \ldots, f_{n}$ arranged from low to high. As the foundation is impacted by instantaneous acceleration, the maximum response amplitude $G_{1}, G_{2}, G_{3}, \ldots, G_{n}$ of each spring vibrator is obtained. Then, the relation curve between the maximum response amplitude $G_{n}$ and the natural frequency of the spring oscillator $f_{n}$ is drawn, and the shock spectrum of the spring oscillator system under the impact of the instantaneous acceleration is obtained.

There are the following relationships among the displacement spectrum, velocity spectrum, and acceleration spectrum in frequency domain [27]:

$$
\begin{aligned}
& V(\omega)=\omega D(\omega), \\
& A(\omega)=\omega^{2} D(\omega) .
\end{aligned}
$$

Because of the above relations among displacement spectrum, velocity spectrum, and acceleration spectrum, three kinds of spectrum values can be represented by one graph, namely, the so-called four parameter logarithmic shock spectrum. The abscissa is the logarithmic form of frequency, the ordinate is the logarithmic form of spectral velocity, the upper left 45 degrees is the logarithmic form of spectral displacement, and the upper right 45 degrees is the logarithmic form of spectral acceleration. For any point in the figure, the displacement spectrum, velocity spectrum, and acceleration spectrum all correspond to each other, as shown in Figure 13.

When calculating the response of spring vibrator, in order to be consistent with the actual motion state of the equipment, the influence of damping should be considered. The introduction of damping reduces the sensitivity of structural response to frequency. Among them, damping has a relatively small impact on the structural response in the 


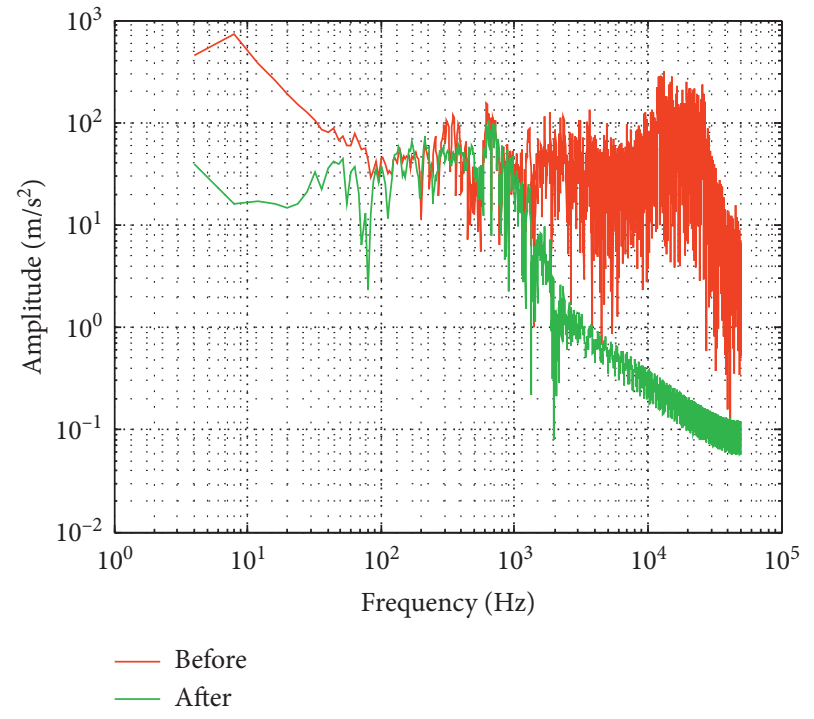

FIGURE 12: Fourier spectrum comparison of signal before and after preprocessing.

low- and high-frequency range but a relatively large impact on the spectral velocity in the intermediate frequency range. Considering the material characteristics of ship equipment, the damping ratio is generally used to draw the shock environment of ship equipment [28].

If the shock signal is expressed in the frequency range, the displacement excitation of the foundation is the main one in the low-frequency range, the acceleration excitation of the foundation is the main one in the high frequency range, and the velocity impact of the foundation is the main one in the medium frequency range. Based on this rule, the shock response spectrum is usually rounded into a design spectrum represented by a fold line. The specific steps of converting the original calculated shock spectrum to the design shock spectrum are as follows:

(1) The curvature below $4 \mathrm{~Hz}$ is not taken into account in the low-frequency area, and the peak value of shock spectrum is taken as the reference, while the valley value of shock spectrum is taken as the reference in the medium frequency area and high-frequency area

(2) The standard design shock spectrum is taken as 0.8 times of the standard design shock spectrum in the low-frequency area, and the standard design shock spectrum is taken as the reference in the medium frequency area and high-frequency area

(3) In case of obvious spectrum drop in the intermediate frequency area or high frequency area, the standard design shock spectrum shall be drawn by 1.5 times of the reference design shock spectrum value, and the following principles shall be followed

(a) If the calculated shock spectrum value is continuously lower than the standard design shock spectrum by more than one octave, the standard design shock spectrum value in this frequency band shall be reduced until the calculated shock

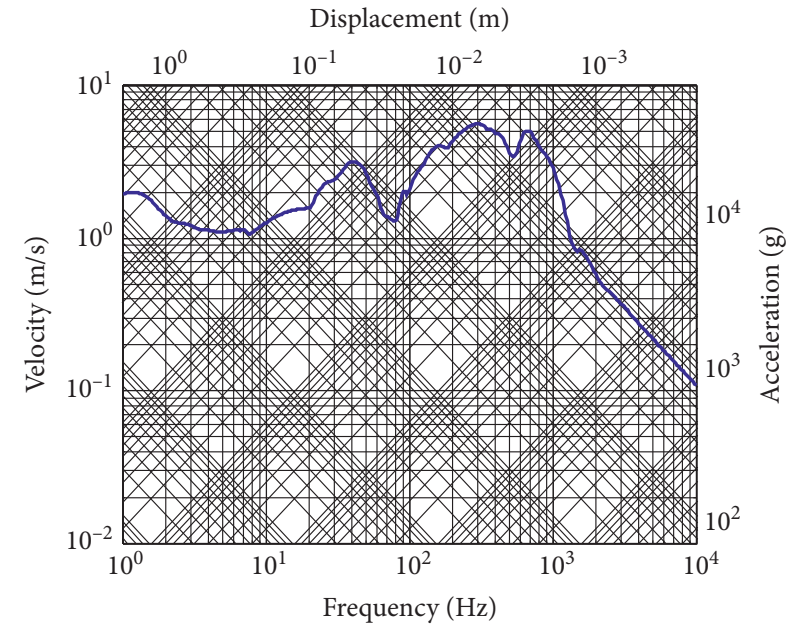

FIGURE 13: Four-parameter logarithmic shock response spectrum.

spectrum value is continuously lower than the standard design shock spectrum by a frequency band equal to one octave

(b) If the area above the standard design shock spectrum is smaller than the area below the standard design shock spectrum, reduce the standard design shock spectrum value of this frequency band until the area above and below the standard design shock spectrum is equal

The design shock spectrum obtained by rounding according to the above method is shown in Figure 14. The flow chart of the acceleration signal processing method described in this paper is shown in Figure 15.

\section{Results and Discussion}

3.1. Working Condition and Measuring Point Position. The working condition of the example is consistent with that of the rigid body in Section 2.2.2. According to the test requirements of the FSP, 11 acceleration measuring points are arranged in three directions of XYZ of the bottom plate in the platform. $X$ direction is along the length of the ship, $y$ direction is along the width of the ship, and $z$ direction is along the depth of the ship. The positions of the acceleration measuring points are shown in Figure 16, and the directions are shown in Table 2.

3.2. Signal Processing Results. According to the above acceleration signal processing method, a total of 29 data of 11 measuring points under the current working condition are processed. The shock response spectrum and design spectrum of channels 6 and 9 are shown in Figure 17:

It can be seen from Figure 17 that the acceleration signal processing method described above has basically eliminated the low-frequency trend component in the signal, which can be rounded into the design spectrum, providing an important basis for subsequent impact environment analysis. The spectral velocity of channel 6 design spectrum is $2.59 \mathrm{~m} / \mathrm{s}$ 


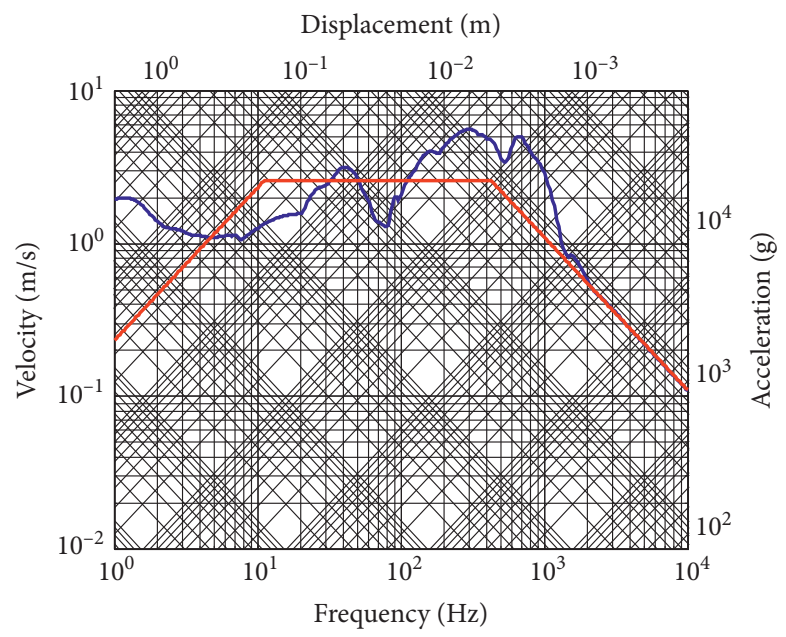

Figure 14: Shock response spectrum and design response spectrum.

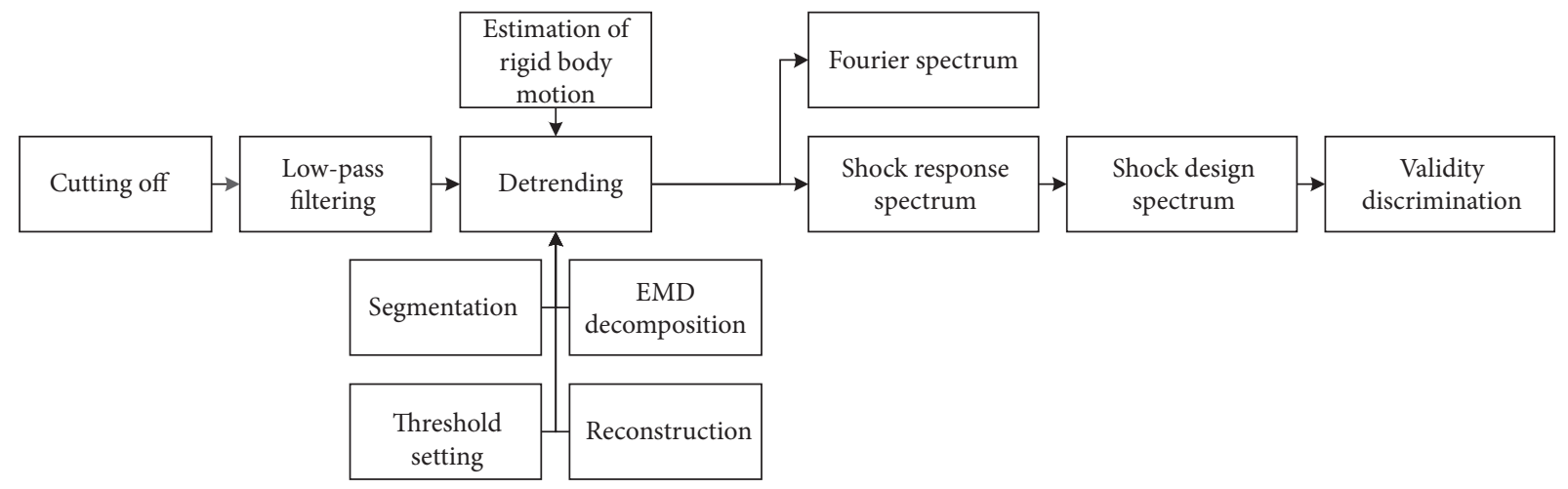

FIGURE 15: Flow chart of the acceleration signal processing method.

and channel 9 design spectrum is $1.85 \mathrm{~m} / \mathrm{s}$, which reflects the difference of design spectral values of different measuring points in the inner bottom.

The comparison of spectral velocity with or without rigid body motion correction is shown in Table $3 . V_{1}$ represents the uncorrected spectral velocity, and $V_{2}$ represents the corrected spectral velocity.

As shown in Table 3, it can be seen that the effect of rigid body correction on the spectral velocity is caused by the change of shock response spectrum caused by rigid body correction, so the design spectral value obtained by rounding the shock response spectrum will also be affected to some extent.

The design spectrum values of all channels on the inner bottom are shown in Table 4:

According to the data in Figure 17 and the position of the measuring points in Table 4 , it can be found that the data of some measuring points in the same direction deviate from the average value obviously, which shows that after the acceleration signal processing, all the data of the measuring points cannot be guaranteed to be effective. In the statistical analysis of the impact environment of the inner bottom, it is necessary to eliminate the invalid measurement point data.
3.3. Validity Discrimination. In the field of engineering, the commonly used method to remove outliers is the Pauta criterion. Now, its principle and application in this paper are briefly described as follows.

For a group of data $x_{i}$, the arithmetic mean is $\bar{x}$, the standard deviation is $\sigma$, and the residual error of each data is $v_{i}=x_{i}-\bar{x}$. If the residual error $v_{b}$ of a measurement value $x_{b}$ satisfies,

$$
\left|v_{b}\right|=\left|x_{b}-\bar{x}\right| \geq 3 \sigma
$$

The bad value $x_{b}$ with gross error value should be eliminated.

In this paper, the spectral displacement and spectral velocity are used as the indexes to test the validity of the data. When both of them pass the test of the Pauta criterion, the data of the measuring point are considered to be true and effective. The reason why the spectral acceleration is not chosen as the validity index is that the value of the spectral acceleration is greatly affected by the filtering, while the left and right frequencies are not directly calculated from the measured data, but from the design spectral value. In addition, in order to solve the problem that Pauta criterion is 


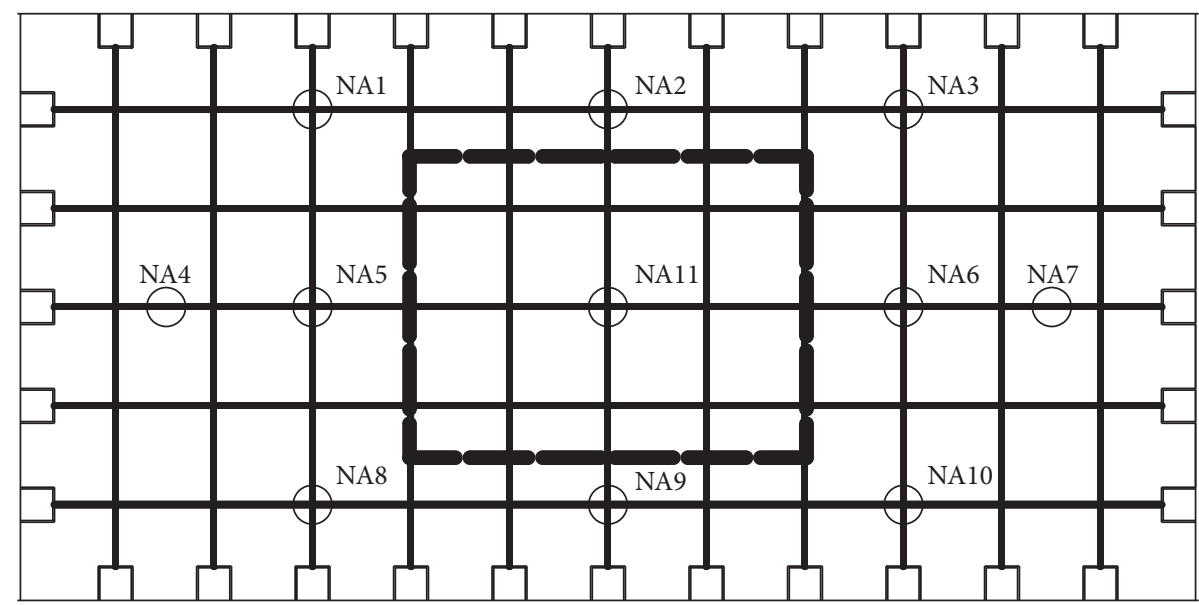

FIGURE 16: Layout of acceleration measuring points.

TABLE 2: Direction statistics of acceleration measuring points.

\begin{tabular}{|c|c|c|c|c|c|}
\hline Channel & Base number & Sensor number & Channel & Base number & Sensor number \\
\hline 1 & NA1 & NA1-X & 16 & NA6 & NA6-Z \\
\hline 2 & & NA1-Y & 17 & NA7 & NA7-Z \\
\hline 3 & & NA1-Z & 18 & NA8 & NA8-X \\
\hline 4 & NA2 & NA2-X & 19 & & NA8-Y \\
\hline 5 & & NA2-Y & 20 & & NA8-Z \\
\hline 6 & & NA2-Z & 21 & NA9 & NA9-X \\
\hline 7 & NA3 & NA3-X & 22 & & NA9-Y \\
\hline 8 & & NA3-Y & 23 & & NA9-Z \\
\hline 9 & & NA3-Z & 24 & NA10 & NA10-X \\
\hline 10 & NA4 & NA4-Z & 25 & & NA10-Y \\
\hline 11 & NA5 & NA5-X & 26 & & NA10-Z \\
\hline 12 & & NA5-Y & 27 & NA11 & NA11-X \\
\hline 13 & & NA5-Z & 28 & & NA11-Y \\
\hline 14 & NA6 & NA6-X & 29 & & NA11-Z \\
\hline 15 & & NA6-Y & & & \\
\hline
\end{tabular}

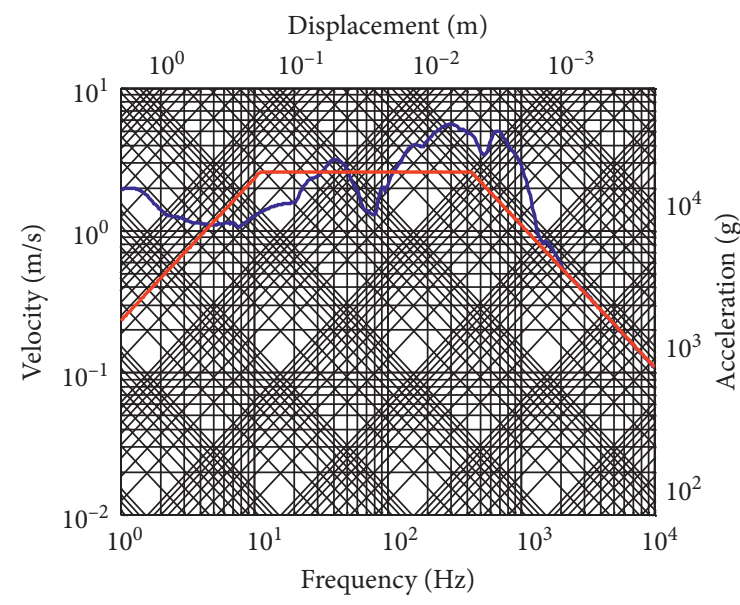

(a)

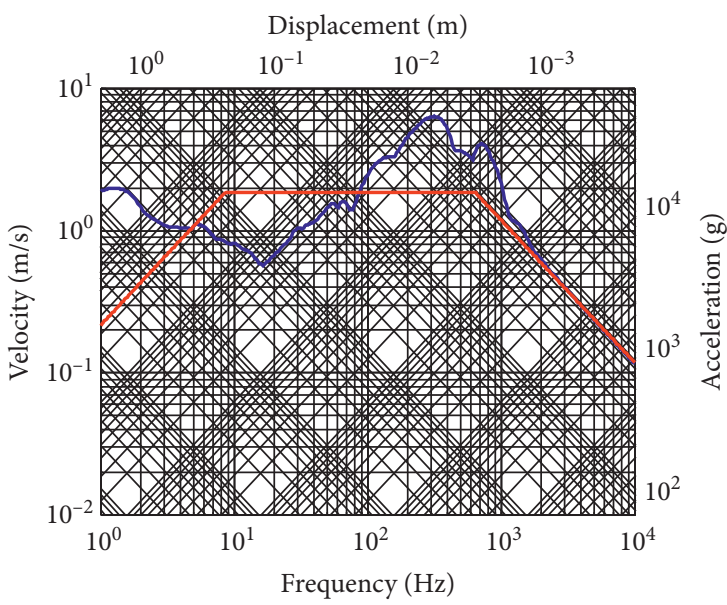

(b)

FIGURE 17: Shock response spectrum and design spectrum of typical channels. (a) Channel 6. (b) Channel 9. 
TABLE 3: The comparison of spectral velocity with or without rigid body motion correction.

\begin{tabular}{lccccc}
\hline Number & $V_{1}(\mathrm{~m} / \mathrm{s})$ & $V_{2}(\mathrm{~m} / \mathrm{s})$ & Number & $V_{1}(\mathrm{~m} / \mathrm{s})$ & $V_{2}(\mathrm{~m} / \mathrm{s})$ \\
\hline 1 & 0.76 & 0.76 & 14 & 1.10 & 1.10 \\
2 & 2.47 & 2.95 & 15 & 1.91 & 3.79 \\
3 & 10.05 & 10.05 & 16 & 2.44 & 2.59 \\
4 & 0.43 & 0.43 & 17 & 2.20 & 2.25 \\
5 & 1.13 & 3.80 & 18 & 0.92 & 0.92 \\
6 & 3.00 & 2.59 & 19 & 2.07 & 3.23 \\
7 & 1.21 & 1.21 & 20 & 2.44 & 2.89 \\
8 & 2.05 & 3.36 & 21 & 0.58 & 4.58 \\
9 & 1.94 & 1.85 & 22 & 3.00 & 2.15 \\
10 & 2.09 & 2.57 & 24 & 1.01 & 1.01 \\
11 & 0.96 & 0.96 & 25 & 3.80 & 3.95 \\
12 & 1.64 & 4.82 & 26 & 2.33 & 2.78 \\
13 & 2.52 & 2.33 & & & \\
\hline
\end{tabular}

TABLE 4: Design spectrum value of each measuring point of inner bottom.

\begin{tabular}{|c|c|c|c|c|c|}
\hline Number & Displacement $(\mathrm{cm})$ & Velocity $(\mathrm{m} / \mathrm{s})$ & Acceleration (g) & Left frequency $(\mathrm{Hz})$ & Right frequency $(\mathrm{Hz})$ \\
\hline 1 & 2.17 & 0.76 & 313.14 & 5.59 & 640.69 \\
\hline 2 & 11.75 & 2.95 & 369.84 & 4.00 & 195.29 \\
\hline 3 & 5.12 & 10.05 & 2970.03 & 31.21 & 461.08 \\
\hline 4 & 1.45 & 0.43 & 115.70 & 4.66 & 424.97 \\
\hline 5 & 15.10 & 3.80 & 370.13 & 4.00 & 152.14 \\
\hline 6 & 3.69 & 2.59 & 707.64 & 11.16 & 426.31 \\
\hline 7 & 1.40 & 1.21 & 385.44 & 13.70 & 498.24 \\
\hline 8 & 13.35 & 3.36 & 328.41 & 4.01 & 152.42 \\
\hline 9 & 3.44 & 1.85 & 763.60 & 8.56 & 643.44 \\
\hline 10 & 7.92 & 2.57 & 689.52 & 5.17 & 418.34 \\
\hline 11 & 1.91 & 0.96 & 467.94 & 7.96 & 764.57 \\
\hline 12 & 19.18 & 4.82 & 616.83 & 4.00 & 199.45 \\
\hline 13 & 3.53 & 2.33 & 1216.55 & 10.52 & 812.85 \\
\hline 14 & 3.52 & 1.10 & 267.80 & 4.96 & 380.80 \\
\hline 15 & 15.05 & 3.79 & 579.05 & 4.01 & 238.37 \\
\hline 16 & 2.45 & 2.59 & 1076.49 & 16.81 & 648.51 \\
\hline 17 & 6.23 & 2.25 & 708.13 & 5.75 & 490.42 \\
\hline 18 & 1.30 & 0.92 & 370.46 & 11.19 & 631.76 \\
\hline 19 & 12.81 & 3.23 & 744.17 & 4.01 & 359.77 \\
\hline 20 & 8.85 & 2.89 & 759.01 & 5.19 & 409.82 \\
\hline 21 & 1.73 & 0.58 & 180.65 & 5.33 & 487.82 \\
\hline 22 & 16.46 & 4.15 & 659.30 & 4.01 & 248.11 \\
\hline 23 & 4.11 & 2.69 & 634.95 & 10.42 & 368.05 \\
\hline 24 & 2.88 & 1.01 & 508.80 & 5.59 & 784.15 \\
\hline 25 & 8.59 & 3.95 & 671.53 & 7.31 & 265.48 \\
\hline 26 & 8.44 & 2.78 & 762.66 & 5.25 & 427.72 \\
\hline
\end{tabular}

TABLE 5: Average value of design spectrum in all directions of inner bottom plate.

\begin{tabular}{lccccc}
\hline Direction & Displacement $(\mathrm{cm})$ & Velocity $(\mathrm{m} / \mathrm{s})$ & Acceleration $(\mathrm{g})$ & Left frequency $(\mathrm{Hz})$ & Right frequency $(\mathrm{Hz})$ \\
\hline$X$ & 2.045 & 0.869 & 326.241 & 7.37 & 576.628 \\
$Y$ & 14.034 & 3.755 & 542.408 & 4.419 & 226.378 \\
$Z$ & 5.407 & 2.505 & 813.171 & 8.759 & 516.162 \\
\hline
\end{tabular}

not applicable due to insufficient data, an empirical threshold can be added, that is, the data beyond the threshold are considered invalid. The threshold value used in this paper is $8 \mathrm{~m} / \mathrm{s}$.

Through the effectiveness analysis, the effective data of measuring points are selected and the average value of design spectrum in each direction of inner bottom are shown in Table 5:

It can be seen from Table 5 that the maximum spectral displacement and velocity in $Y$ direction of inner base plate are $14.034 \mathrm{~cm}$ and $3.755 \mathrm{~m} / \mathrm{s}$, respectively; the second is the spectral displacement and velocity in $Z$ direction, which are 
$5.407 \mathrm{~cm}$ and $2.505 \mathrm{~m} / \mathrm{s}$, respectively; the least is the spectral displacement and velocity in $X$ direction, which are $2.045 \mathrm{~cm}$ and $0.869 \mathrm{~m} / \mathrm{s}$, respectively. The main reason for the difference in the design spectrum values in different directions is the angle of attack of the working condition, which is a positive and horizontal working condition. The distance between the charge and the side of the ship is $6.71 \mathrm{~m}$, and the distance between the charge and the free liquid level is $8.2 \mathrm{~m}$. In the actual test, the instantaneous position of the charge initiation may deviate from the preset position due to the action of water flow, so there is also a response in the $X$ direction.

\section{Conclusions}

Due to the severe shock environment and the measurement capability of current test system in underwater explosion research field, the acceleration signal processing method is an important step to obtain relatively accurate test results. The existing methods cannot distinguish the rigid body motion response of FSP and the real trend term when eliminating the low-frequency trend term. To address this problem, this paper proposed a complete set of acceleration signal processing method in which the rigid body motion revision is included. In the estimation of FSP rigid body motion, spherical wave theory is used to describe the shock wave generated by underwater explosion. The empirical formula of Zamyshlyayev is used for shock wave model. G-H spherical bubble theory is used for bubble model. KrylovFroude hypothesis is used to calculate the structural load. In addition, the signal is processed by low-pass filtering in frequency domain to eliminate high-frequency noise produced in the test process. This method can effectively eliminate the low-frequency trend component and high-frequency noise component in the signal and has good robustness.

Through the research of this paper, the following conclusions are drawn:

(1) All kinds of interferences, including low-frequency trend term and high-frequency noise component, are unavoidable in the acceleration response data measured in underwater explosion shock test of FSP, which must be removed before shock spectrum analysis.

(2) The segmented EMD method can eliminate the lowfrequency trend term in the signal, but it cannot recognize the rigid body motion signal in the lowfrequency range.

(3) The rigid body motion model of the FSP based on the KrylovFroude hypothesis, shock wave empirical formula and G-H spherical bubble model are adopted to simulate the rigid body motion response of the FSP subject to the underwater explosion. The rigid body motion response can be modified as the measured signal to get more accurate real response signal.

(4) The data validity judgment method based on Pauta criterion can screen out invalid data accurately, which leads to a more accurate assessment of FSP shock environment.

\section{Data Availability}

The data used to support the findings of this study are included within the article.

\section{Conflicts of Interest}

The authors declare that there are no conflicts of interest regarding the publication of this paper.

\section{Acknowledgments}

This work was supported by the Equipment Preresearch Foundation (61400020302), National Natural Science Foundation of China (11602069), National Science and Technology Major Project (2017-V-0002-0051), and Natural Science Foundation of Heilongjiang Province of China (QC2018052).

\section{References}

[1] L. Zhang, Z. Du, J. Wu, Ji Chen, C. Zhang, and L. Feng, "Lowfrequency shock response data analysis of underwater explosion test of 200-ton class floating shock platform," Chinese Journal of Ship Research, vol. 13, no. 3, pp. 60-65, 2018, in Chinese.

[2] J. Wang and X. Hu, The Application of MATLAB in Vibration Signal Processing 2006, China Water Conservancy and Hydropower Press, Intellectual Property Press, Beijing, China, in Chinese.

[3] Q. Wang, Study on the Error Correction Method of Trend Term of Underwater Explosion Shock Acceleration Signal, Shenyang University of Technology, Shenyang, China, 2017, in Chinese.

[4] J. Chen and S. Wang, "An improved wavelet correction method for zero drift of impact acceleration," Mine Warfare and Ship Self-Defence, vol. 4, pp. 18-23, 2012, in Chinese.

[5] Z. Wu, C. Wang, and A. Ren, "Optimal selection of wavelet base functions for eliminating signal trend based on wavelet analysis," Transactions of Beijing Institute of Technology, vol. 33, no. 8, pp. 811-814, 2013, in Chinese.

[6] P. C. Chu, C. Fan, and N. Huang, "Compact empirical mode decomposition: an algorithm to reduce mode mixing, end effect, and detrend uncertainty," Advances in Adaptive Data Analysis, vol. 4, no. 3, Article ID 1250017, 2012.

[7] L. Yuan, Q. Xie, M. Zhong, L. Lu, and X. Li, "Research on trend removing methods in preprocessing analysis of blasting vibration monitoring signals," Engineering Mechanics, vol. 29, no. 10, pp. 63-68, 2012, in Chinese.

[8] P. Singh, "Nonpolynomial spline based empirical mode decomposition," in Proceedings of the 2013 International Conference on Signal Processing and Communication (ICSC), Noida, India, December 2013.

[9] KAS, A Mathematical Model of Transient Motion Response of the US Navy Floating Platform, The University of Akron, Akron, Ohio, 1984.

[10] B. V. Zamyshlyayev, Dynamic Loads in Underwater Explosion, Naval Intelligence Support Center, Washington, DC, USA, 1973.

[11] L. Geers and K. S. Hunter, "An integrated wave-effects model for an underwater explosion bubble," in Proceedings of the 
Acoustical Society of America, p. 111, Pennsylvania, PA, USA, June 2002.

[12] T. L. Geers and C.-K. Park, "Optimization of the G\&H bubble model," Shock and Vibration, vol. 12, no. 1, pp. 3-8, 2005.

[13] Y. Lu, Ship Seakeeping, Shanghai Jiaotong University Press, Shanghai, China, 1985, in Chinese.

[14] J. Du, Y. Li, Z. Du, and H. Li, "Investigation of input loading for shipboard equipment in shock test," Acta Armamentarii, vol. s1, pp. 78-86, 2015, in Chinese.

[15] L. Zeng, C. Shang, and S. Wang, "Effects of underwater explosion bubble on shock environment of warship," Chinese Journal of Ship Research, vol. 3, pp. 66-71, 2018, in Chinese.

[16] H. A. Gaberson, Shock Data Filtering Consequences, pp. 961-977, Springer, New York, NY, USA, 2011.

[17] G. Wang, Y. Liu, X. Jin, and Y. He, “Trend term processing based on least square principle and its matlab implementation," Colored Equipment, vol. 5, pp. 4-8, 2005, in Chinese.

[18] S. Tang, A Vibration Signal Acquisition and Analysis System Based on MATLAB, Ocean University of China, Qingdao, China, 2007, in Chinese.

[19] D. Li, X. Xiong, and B. Li, "Discussion on vibration acceleration signal processing," Electromechanical Engineering Technology, vol. 37, no. 9, pp. 50-52, 2008, in Chinese.

[20] C. Wang and $\mathrm{Z}$. Wu, "Reasearch on the method of the preferring of wavelet based for eliminating the signal trend of vehicle random vibration," in Proceedings of the 2012 Academic Annual Meeting of off Road Vehicle Technology Branch of China Automotive Engineering Society, Wuhan, China, 2012, in Chinese.

[21] X. Zhu and N. Han, "Removal of non-stationary signal trend items by empirical mode decomposition," Journal of Spacecraft TT\&C Technology, vol. 31, no. 1, pp. 65-70, 2012, in Chinese.

[22] B. Liang and T. Wang, "Method of vibration signal trend extraction based on HHT," Electronic Measurement Technology, vol. 36, no. 2, pp. 119-122, 2013, in Chinese.

[23] H. Li, H. Pan, and H. Ren, "Baseline correction of impact signals using the cross-correlation coefficient of shock response spectrum," Shock and Vibration, vol. 35, no. 16, pp. 219-225, 2016, in Chinese.

[24] D. O. Smallwood and J. S. Cap, "Salvaging transient data with overloads and zero offsets," Office of Scientific \& Technical Information, Oak Ridge, TN, USA, Tech. Rep, 1997.

[25] N. E. Huang and S. S. P. Shen, Hilbert-huang Transform and its Applications, World Scientific, Singapore, Singapore, 2nd edition, 2014.

[26] J. Wang, Research on the Impact Dynamic Charecteristics of the Floating Shock Platform, Harbin Engineering University, Harbin, China, 2015, in Chinese.

[27] Yu Wang and H. Hua, Theory and Application of Modern Ship Impact, Science Press, Beijing, China, 2005, in Chinese.

[28] L. H. Feng, Y. Wang, J. Du, and J. Chen, "Research on equivalency of shock response calculation methods for equipments on board," Ship Engineering, vol. 33, pp. 210-214, 2011, in Chinese. 